\title{
DNA mismatch repair gene MLH1 induces apoptosis in prostate cancer cells
}

\author{
Shinichiro Fukuhara ${ }^{1,2,3, *}$, Inik Chang ${ }^{1,4, *}$, Yozo Mitsui 1,2,5, Takeshi Chiyomaru ${ }^{1,2,6}$, \\ Soichiro Yamamura ${ }^{1,2}$, Shahana Majid ${ }^{1,2}$, Sharanjot Saini ${ }^{1,2}$, Hiroshi Hirata ${ }^{1,2}$, Guoren \\ Deng $^{1,2}$, Ankurpreet Gill ${ }^{1}$, Darryn K. Wong ${ }^{1}$, Hiroaki Shiina ${ }^{5}$, Norio Nonomura ${ }^{3}$, \\ Rajvir Dahiya ${ }^{1,2}$ and Yuichiro Tanaka ${ }^{1,2}$ \\ ${ }^{1}$ Department of Surgery/Urology, Veterans Affairs Medical Center, San Francisco, California, United States of America \\ 2 Department of Urology, University of California, San Francisco, California, United States of America \\ ${ }^{3}$ Department of Urology, Osaka University Graduate School of Medicine, Suita, Japan \\ ${ }^{4}$ Department of Oral Biology, Yonsei University College of Dentistry, Seoul, Korea \\ ${ }^{5}$ Department of Urology, Shimane University Faculty of Medicine, Izumo, Japan \\ ${ }^{6}$ Department of Urology, Kagoshima University Graduate School of Medical and Dental Sciences, Kagoshima, Japan \\ * These authors contributed equally to this work
}

Correspondence to: Yuichiro Tanaka, email: yuichiro.tanaka@ucsf.edu

Keywords: MLH1, C-Abl, apoptosis, prostate cancer

Received: May 29, $2014 \quad$ Accepted: August 05, 2014 Published: August 06, 2014

This is an open-access article distributed under the terms of the Creative Commons Attribution License, which permits unrestricted use, distribution, and reproduction in any medium, provided the original author and source are credited.

\section{ABSTRACT}

Mismatch repair (MMR) enzymes have been shown to be deficient in prostate cancer (PCa). MMR can influence the regulation of tumor development in various cancers but their role on PCa has not been investigated. The aim of the present study was to determine the functional effects of the mutL-homolog 1 (MLH1) gene on growth of PCa cells. The DU145 cell line has been established as MLH1-deficient and thus, this cell line was utilized to determine effects of MLH1 by gene expression. Lack of MLH1 protein expression was confirmed by Western blotting in DU145 cells whereas levels were high in normal PWR-1E and RWPE-1 prostatic cells. MLH1-expressing stable transfectant DU145 cells were then created to characterize the effects this MMR gene has on various growth properties. Expression of MLH1 resulted in decreased cell proliferation, migration and invasion properties. Lack of cell growth in vivo also indicated a tumor suppressive effect by MLH1. Interestingly, MLH1 caused an increase in apoptosis along with phosphorylated c-Abl, and treatment with MLH1 siRNAs countered this effect. Furthermore, inhibition of c-AbI with STI571 also abrogated the effect on apoptosis caused by MLH1. These results demonstrate MLH1 protects against PCa development by inducing C-Abl-mediated apoptosis.

\section{INTRODUCTION}

Prostate cancer $(\mathrm{PCa})$ is the most frequently diagnosed malignancy and the second leading cause of cancer death among males in the United States [1]. It is estimated that in 2014, there will be 233,000 new cases and 29,480 deaths due to $\mathrm{PCa}$ [1]. PCa is a disease of aging as 1 in 43 of those aged 50 to 59, 1 in 16 aged 60 to 69 , and 1 in 9 aged 70 years and older will develop invasive disease [1]. Most cases of PCa are treatable with androgen-deprivation therapy however the majority of $\mathrm{PCa}$ recur as androgen-independent, metastatic disease that leads to death within several years. Currently, no effective therapies are available to cure androgen-independent $\mathrm{PCa}$. Thus, new prognostic markers and effective treatment strategies are urgently needed.

A factor that may prevent the prostate carcinogenesis process is the DNA mismatch repair (MMR) system that consists of various types of proteins such as the MutS homologues and MutL homologues (MLH) [2, 3]. Defects in this MMR pathway result in an increased rate of mutation or genetic instability, which in turn leads 
to defects in genes that regulate cell proliferation and death [4], thereby increasing susceptibility to cancers. Alternatively, MMR genes have also been shown to sensitize cells toward DNA lesions and trigger apoptosis, cell cycle arrest, and cell death $[5,6]$. Thus, MMR is essential for proper cellular function and health of the individual.

Clinical case reports have shown that PCa can occur in men afflicted with hereditary non-polyposis colorectal cancer (HNPCC), a disease known for mutations in MMR $[7,8]$. The cumulative lifetime risk of PCa was calculated to be two-fold higher among individuals with HNPCC and defective MMR as compared to the general population [8]. In a study by Grindedal et al [9], nine individuals contracted PCa among a cohort of 106 Norwegian men described as carriers or obligate carriers of MMR mutation and this rate was much higher than the 1.52 expected to occur by chance in the Norwegian population $(P<0.01)$. These men were younger at the time of diagnosis $(60.4$ years vs. 66.6 years, $P=0.006$ ) and had higher Gleason scores of 8 to 10 compared to men diagnosed prior to 70 years of age in the population $(P<0.00001)$. KaplanMeier analysis revealed that the cumulative risk of PCa diagnosis by age 70 years was $30 \%$ in MMR gene mutation carriers compared to $8 \%$ in the population. Thus MMR imperfections can lead to PCa.

Among the various MMR genes, hundreds of mutations and polymorphisms have been identified and interestingly, most of these variants are observed in the MLH1 gene $(50 \%)$, thus making this a highly susceptible gene in the carcinogenesis process [10]. Defects in MLH1 have been documented in various cultured prostate cell lines. Among the first reports was the PCa line DU145 where Boyer et al [11] identified a mutation in the MLH1 gene after demonstrating reduced MMR activity and microsatellite instability. Chen et al [12] confirms genomic instability in DU145 cells where MLH1 protein expression was lacking. A prior study from our laboratory [13] show the DU145, LNCaP, and PC3 PCa cell lines to have much lower levels of MLH1 protein expression and these all had reduced DNA repair activity as compared to the MMR proficient Hela cells.

MLH1 expression has also been studied in prostate tissue. A report by Chen et al [12] found that in the normal prostate gland, MLH1 protein was predominantly detected in the nuclei of glandular luminal epithelium, basal cells, and some stromal cells. This pattern of MLH1 expression was also observed in the normal adjacent region of prostate tumor tissue. In malignant prostate cells however, MLH1 levels were found to be less than that observed in normal adjacent areas. Other studies also show reduced MLH1 protein expression in prostate tumor regions as compared to normal adjacent [14, 15]. Additionally, MLH1 expression was found to be much lower in $\mathrm{PCa}$ when compared to benign prostatic hyperplasia tissue [15].

The MLH1 gene is thus shown to be reduced in PCa cell lines and tissues. Therefore in this report, we characterize the functional role the MLH1 gene plays in PCa cells. Our results are the first to show that re-expressing the MLH1 gene in PCa cells causes inhibition of cell growth both in vitro and in vivo. Also, we demonstrate MLH1 to enhance apoptosis and induce phosphorylation of the $\mathrm{c}-\mathrm{Abl}$ protein as a mechanism for its protective role in PCa cells.

\section{RESULTS:}

\section{Prostate cancer cell line DU145 is MLH1 deficient}

Prior studies have established DU145 cells to be MLH1-deficient $[12,13]$ and thus, we selected this cancerous cell line for further studies. Initially, we confirmed protein and RNA expression levels of MLH1 in DU145 cells and as a comparison, measured expression in the normal prostate epithelial cell lines, PWR-1E and RWPE-1. Figure 1A shows no protein detected and 1B significantly reduced RNA expression for MLH1 in

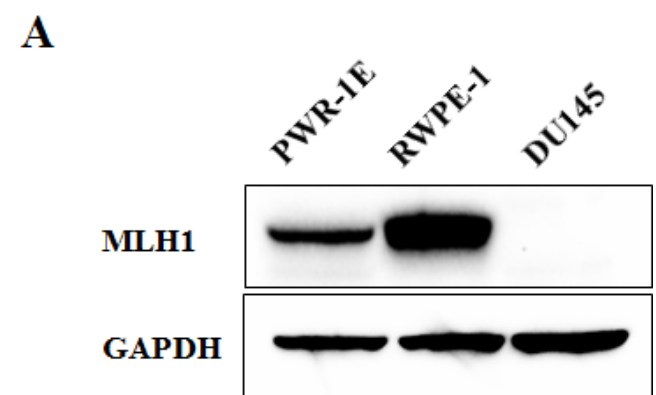

B

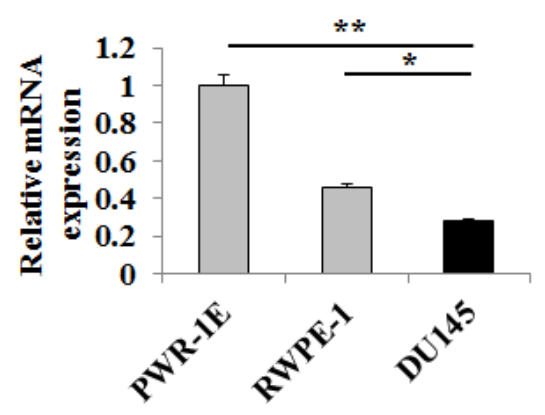

Figure 1: MLH1 expression is downregulated in DU145 PCa cells. Prostatic cell lines were grown in culture dishes for two days. (A) Representative immunoblot displaying MLH1 protein expression in DU145 and normal prostate epithelial PWR-1E and RWPE-1 cells. GAPDH was used as loading control. (B) Relative mRNA expression levels of MLH1 in cell lines as determined by real-time PCR. Expression levels are normalized to PWR-1E. Data are presented as mean \pm SEM of three experiments. Asterisks denote differences between the compared values: $* \mathrm{P}<0.05$, $* * \mathrm{P}<0.01$. 
DU145 cells whereas levels were high in both PWR-1E $(\mathrm{P}<0.01)$ and RWPE-1 $(\mathrm{P}<0.05)$.

\section{Effect of MLH1 re-expression on cell proliferation, migration and invasion}

Absence of MLH1 protein in DU145 cells led us to examine whether re-expressing MLH1 affects the growth of these cells. To do this, we established a DU145 cell line that stably expresses MLH1. As shown in Figure 2A, MLH1 protein was detected by Western blotting in stable MLH1-transfected DU145 cells but not in mock or vector control cells. To determine the effects of MLH1 on cellular properties, we conducted various functional analyses. MTS assay show that cell proliferation was reduced over $30 \%$ after 48 hours in DU145 cells expressing MLH1 compared to vector control $(\mathrm{P}<0.01)$ (Figure $2 \mathrm{~B})$. Wound healing assay demonstrated significant inhibition of cell migration in MLH1-stable compared with vectortransfected DU145 cells after 24 hours (73\% versus 89\% closure, respectively, $\mathrm{P}<0.01$ ) (Figure $2 \mathrm{C}$ ). Matrigel invasion assay also show that the number of invading cells was significantly decreased in MLH1 transfectants with absorbance at $560 \mathrm{~nm}$ of 0.22 compared with vector control having absorbance of $0.28(\mathrm{P}<0.01)$ (Figure 2D). These results indicate MLH1 to play an important role in reducing tumor cell proliferation, migration and invasion.
A

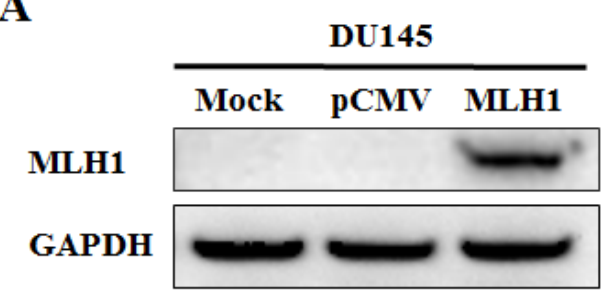

C

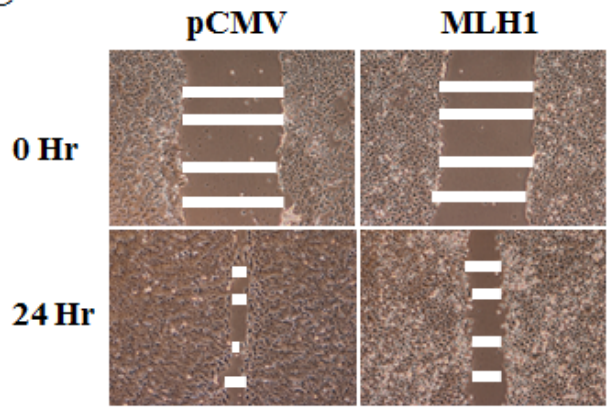

D

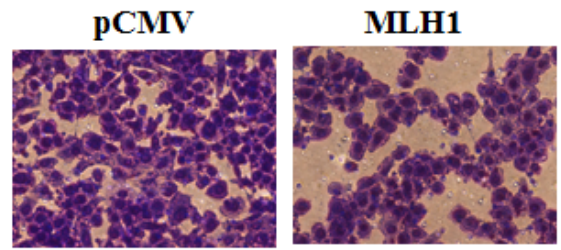

B
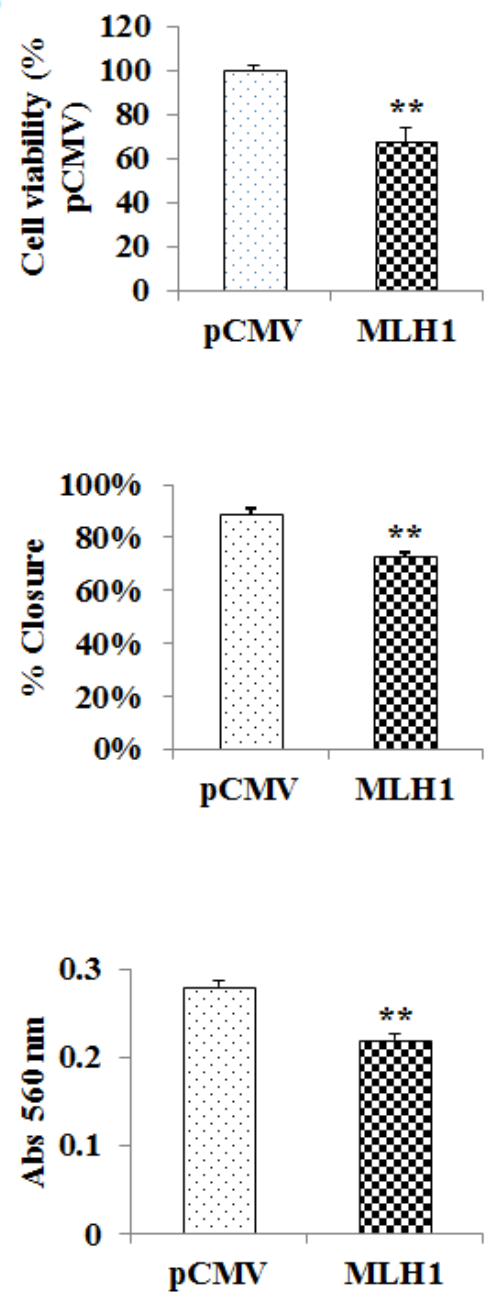

Figure 2: Re-expression and tumor suppressive effect of MLH1 on DU145 cells. (A) Ectopic expression of MLH1. DU145 cells stably transfected with either MLH1 or empty vector (pCMV) along with mock (parental DU145 cells treated with transfection reagent alone) were grown for 48 hours and underwent Western analyses. GAPDH was used as loading control. (B) Cell proliferation as analyzed by the MTS cell proliferation assay 48 hours after plating cells. Results are expressed as \% and normalized to pCMV control. (C) Cell migration as measured by wound healing assay. A wound was formed by scraping culture dishes using a pipet tip and closure measured after 24 hours. Left: Representative images of wound healing assay are shown. Right: Migration expressed as \% closure of wound. (D) Cell invasiveness as measured using Matrigel. Cells were placed onto transwell membrane and allowed to invade for 24 hours. Left: Representative images of invading cells are shown. Right: Cell invasiveness as measured by absorbance (Abs) at $560 \mathrm{~nm}$. Data are presented as mean $\pm \mathrm{SEM}$ of at least three experiments; **P $<0.01 \mathrm{MLH} 1$ versus $\mathrm{pCMV}$. 


\section{Effect of MLH1 expression on tumorigenicity in vivo}

To validate the suppressive effect of MLH1 under in vitro conditions, we also determined effects of MLH1 on tumor growth in animal models. Stable MLH1 and pCMV DU145 cells were subcutaneously injected into nude mice. We observed that expression of MLH1 inhibited DU145 cell tumor formation throughout the duration which lasted 5 weeks whereas by week 4 , tumor growth was visible in pCMV animals. By week five, tumor sizes were dramatically increased to an average of 565 $\mathrm{mm}^{3}$ in controls compared to $13 \mathrm{~mm}^{3}$ in MLH1-treated mice $(\mathrm{P}<0.05)$ (Figure 3$)$. These results suggest MLH1 suppresses $\mathrm{PCa}$ cell growth in vivo.

\section{MLH1 influences cellular apoptosis}

Since MLH1 restoration significantly inhibits cell growth and progression of DU145 cells both in vitro and in vivo, we hypothesized that its expression may induce apoptosis. Figure 4A shows that the apoptotic and early apoptotic fractions (upper right and lower right quadrants of biparametric histograms, respectively) were increased in MLH1-transfectants compared to vector control after 48 and 72 hours of growth. Total apoptosis levels were 10.3\% versus $15.7 \%$ at 48 hours $(\mathrm{P}<0.05)$ and $14.6 \%$ versus $26.0 \%$ at 72 hours $(\mathrm{P}<0.05)$ for $\mathrm{pCMV}$ versus MLH1expressing cells, respectively (Figure 4A).

\section{pCMV MLH1}



\section{MLH1 induces c-Abl phosphorylation}

The pro-apoptotic role for MLH1 observed suggests that apoptotic pathways are affected. It has been reported that $\mathrm{c}-\mathrm{Abl}$ mediates MLH1-dependent apoptosis in other cell types such as colon cancer cells [16]. Therefore, we examined the expression of c-Abl in DU145 cells and found that phosphorylated c-Abl protein was upregulated in MLH1-transfectants whereas levels of the non-phosphorylated form was not changed (Figure 4B). Additionally, we found that MLH1 expression caused an increase in cleaved PARP which further supports the pro-apoptotic role of MLH1. These results indicate c-Abl phosphorylation to moderate the apoptotic effects of MLH1 in PCa cells.

\section{Inhibition of MLH1 prevents apoptotic cell death in MLH1-expressing cells}

To establish MLH1 as a key effector in the c-Ablmediated apoptotic signaling pathway, we measured apoptotic cell death by exploiting siRNA to selectively knock down MLH1 (siMLH1) in MLH1-stable DU145 cells. We first confirmed the ability of siMLH1 to suppress endogenous MLH1 levels. Administration of three MLH1 siRNAs to MLH1-expressing DU145 cells decreased both basal MLH1 mRNA (data not shown) and protein (Figure 5A) expression. Interestingly, all three siMLH1s also significantly reduced apoptosis (siMLH1-1 (4.6\%, $\mathrm{P}<0.01)$, siMLH1-2 (3.6\%, $\mathrm{P}<0.01)$, siMLH1-3 $(6.4 \%, \mathrm{P}<0.05)$ compared to siRNA negative control (10.1\%) (Figure 5B). Furthermore, MLH1 knockdown


Figure 3: Expression of MLH1 suppresses growth of DU145 cells in vivo. Athymic nude mice were injected subcutaneously with stable MLH1 or pCMV-transfected DU145 cells and growth monitored over time. Left: Representative image of tumors in mice five weeks after injection of cells. Right: Growth of tumor size plotted over time. Data are presented as mean \pm SEM of five mice per group; $* \mathrm{P}<0.05 \mathrm{MLH} 1$ versus $\mathrm{pCMV}$ for each time point. 
A

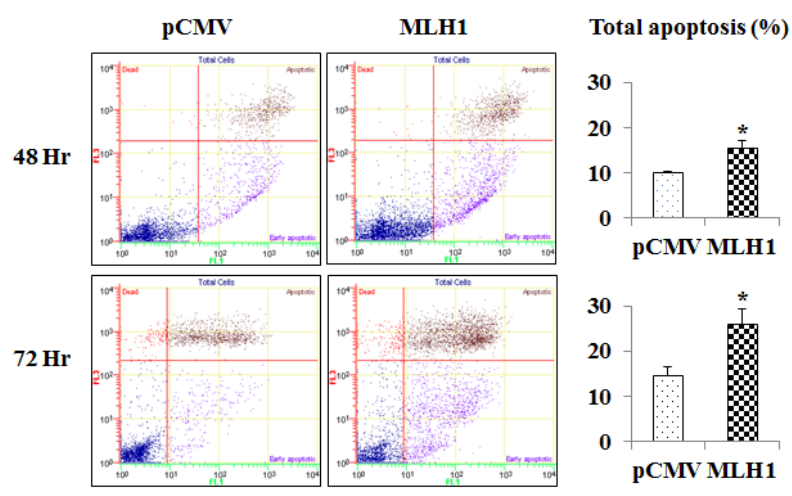

B

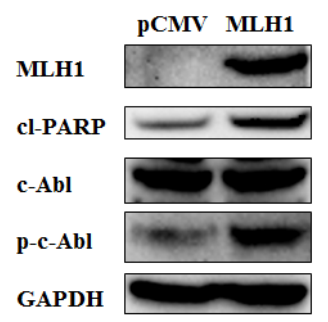

Figure 4: MLH1 expression upregulates apoptosis and phosphorylated c-Abl (p-c-Abl). Stable MLH1-expressing and vector control DU145 cells were grown over time. (A) Apoptosis as measured by flow cytometric analyses. Representative biparametric histogram showing cell population in early (bottom right quadrant) and late (top right quadrant) apoptotic, and viable (bottom left quadrant) states. Left: pCMV control, Middle: MLH1-expressing, Right: Total apoptosis \%, Upper: 48 hours of growth, Lower: 72 hours of growth. Bar graph of total apoptosis \% is presented as mean \pm SEM of three experiments, ${ }^{*} \mathrm{P}<0.05$ MLH1 versus $\mathrm{pCMV}$. (B) Protein levels of MLH1, cleaved PARP (cl-PARP), c-Abl, and p-c-Abl were determined by Western blot analyses after growing pCMV and MLH1 cells for 48 hours. GAPDH was used as loading control.

A

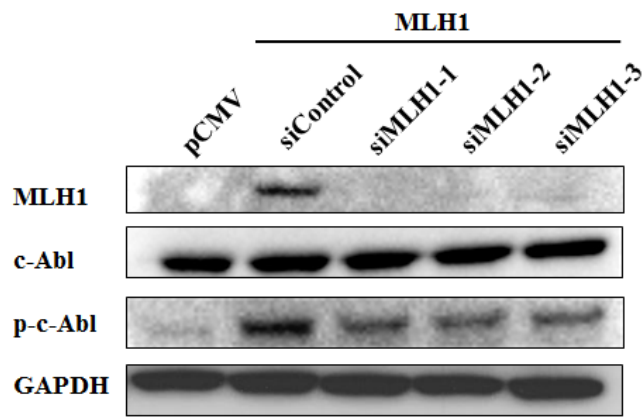

B

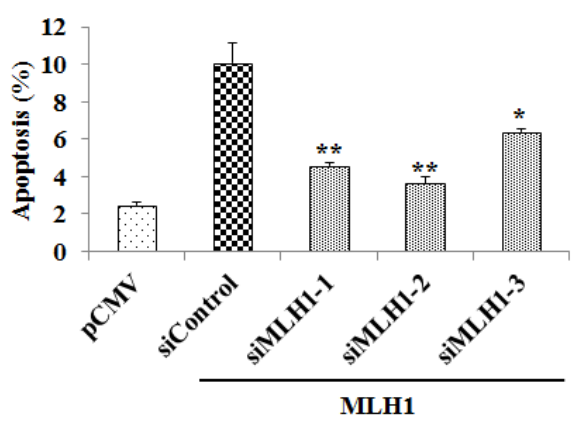

Figure 5: MLH1 knockdown reduces phosphorylated c-Abl (p-c-Abl) and apoptosis. Three MLH1 siRNAs (siMLH1s) were transfected individually along with a non-specific siRNA control (siControl) into stable MLH1-expressing DU145 cells for 48 hours. pCMV control cells treated with Lipofectamine are also included. (A) Protein levels of MLH1, c-Abl, and p-c-Abl in cells as determined by Western blot analyses. GAPDH was used as loading control. (B) Total apoptotic cells were analyzed by flow cytometry. Data are expressed as mean $\pm \mathrm{SEM}$ of three experiments; $* \mathrm{P}<0.05 * * \mathrm{P}<0.01$ siMLH1s versus siControl. 
decreased phosphorylation of c-Abl without affecting nonphosphorylated c-Abl protein levels (Figure 5A). These results demonstrate that MLH1 induces apoptosis by c-Abl phosphorylation in PCa cells.

\section{Inhibition of c-Abl prevents apoptotic cell death in MLH1-expressing cells}

To confirm the role of $\mathrm{c}-\mathrm{Abl}$ as a regulator of $\mathrm{MLH} 1-$ stimulated apoptosis, we examined the effect of the c-Abl inhibitor, STI571. MLH1 causes apoptosis in DU145 cells but when treated with STI571, a marked decrease of apoptosis to vector control levels was observed in MLH1expressing cells $(\mathrm{P}<0.01)$ (Figure 6). We also observe phosphorylated c-Abl to be reduced due to STI571 (data not shown). These results further demonstrate that c-Abl regulates apoptosis in MLH1-expressing DU145 PCa cells.

\section{DISCUSSION}

The MMR system recognizes and corrects misincorporated nucleotides and insertion/deletion mis-pairs formed during DNA replication [2, 3]. Loss of proteins critical in this repair process however, can result in genetic alterations that may ultimately lead to cancer. MMR deficiency also predisposes cells of the body to an increased frequency of inactivating mutations in genes important for suppressing carcinogenesis [4]. Besides their established role in DNA repair, MMR genes are also involved in cell cycle checkpoints and apoptosis stimulated by DNA damage $[5,6]$. Resistance to apoptosis and cell death were shown in cells deficient in MMR genes when treated with DNA damaging chemotherapeutic agents [17, 18]. The MMR system is thus vital for maintaining cell integrity and disease prevention.
Among the various MMR genes, MLH1 has been shown to be among the most essential in sensitizing cells to undergo apoptosis [19]. This gene has also been shown to be more prone to mutation [10]. The genetic status of MLH1 in cells thus has high impact in cellular function. The MLH1 gene is located in chromosome 3 p21 region, consists of 19 exons and is roughly 58 kilobases in length [20]. MLH1 is a nuclear protein that consists of 756 amino acids with a size of $80 \mathrm{kDa}$.

In prostate tissues, MLH1 has been shown to be downregulated in tumor regions as compared to normal prostate [12], normal adjacent regions [12, 14, 15] and benign hyperplasia [15]. Given that MLH1 can affect cell growth parameters, we explored the functional significance the MLH1 gene has on PCa cells which has never been done. MLH1 protein expression in the DU145 cell line has previously been shown to be downregulated $[12,13]$ and our results confirm this lack of protein expression. This reduction is apparently due to a truncation in the MLH1 gene caused by a premature stop codon as observed by Chen et al [12]. These investigators discovered a loss of 5 nucleotides at the start of the coding region of exon 2 that results in a frameshift producing a TGA stop at codon 39. Additionally, we observed that RNA levels are reduced in this cell line which indicates that the transcriptional machinery in DU145 cells is also affected. Thus, DU145 $\mathrm{PCa}$ cells are an ideal cell line to determine functional effects of the MLH1 gene by re-expression.

By expressing the MLH1 gene in DU145 cells, we observed that this gene caused a significant reduction in proliferation, migration, and invasive properties of this PCa cell line. A similar effect was observed in vivo as MLH1-expressing cells inhibited cell growth. This repressive activity was found to be due to apoptosis as MLH1 cells had a much higher rate compared to that of vector control. The apoptotic environment was also

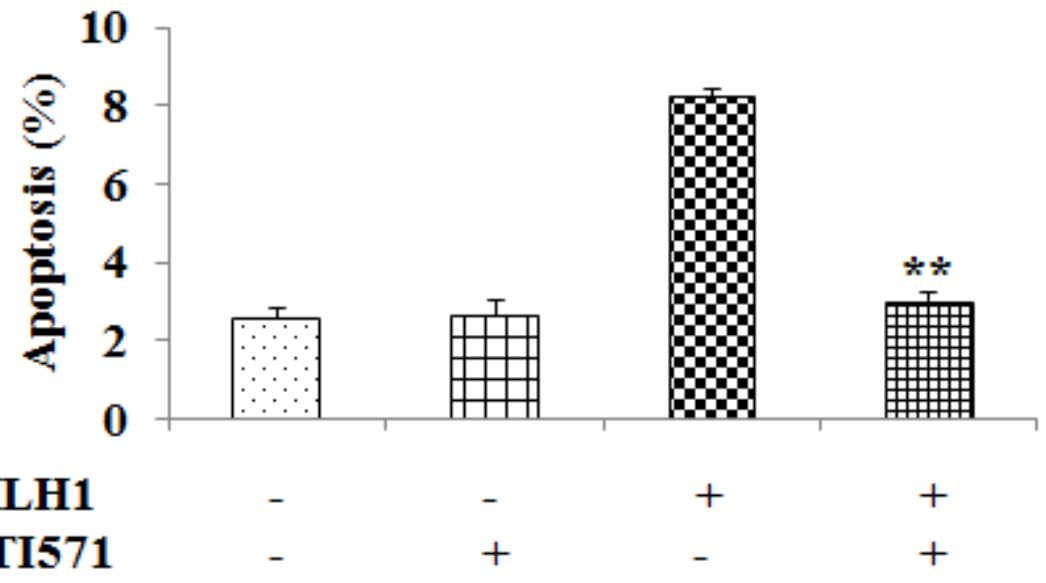

Figure 6: Inhibition of c-Abl nullifies MLH1-triggered apoptotic effect. MLH1-expressing and pCMV control DU145 transfectants were treated with either DMSO or STI571 and maintained for 48 hours. Total apoptotic cells were then analyzed by flow cytometry. Data are expressed as mean \pm SEM of three experiments; **P $<0.01 \mathrm{MLH} 1+/ \mathrm{STI} 571+$ versus MLH1+/STI571-. 
supported by an observed rise in cleaved PARP, suggesting the presence of caspases [21, 22]. On the contrary, silencing MLH1 in normal prostatic PWR-1E cells caused an increase in proliferation for two of three siMLH1 treatments (Supplementary Figure 1). Thus, MLH1 plays a tumor suppressor role in PCa cells. In concordance with our results, MLH1 has been shown by others to play an important role in apoptosis and cell cycle control $[5,6$, 23]. In MLH1-deficient HCT116 colon cancer cells, expressing MLH1 by injecting chromosome 3 led to growth arrest whereas parental HCT116 or HCT116 cells injected with chromosome 2 had no effect after treatment with methylnitronitrosoguanidine (MNNG) [24]. Hawn et al [25] finds these HCT116+chromosome 3 cells to be arrested at G2 phase after treatment with 6-thioguanine or MNNG. In the ovarian cancer cell line A2780MNU1 that is MMR deficient, re-expression of MLH1 by gene transfection resulted in marked reductions of cell survival and proliferation, with higher percentage of cells displaying active caspase 3 after drug treatment [26]. In a report by Zhang et al [19], MLH1 cDNA was expressed in HCT116 and they find apoptosis to be increased nearly 3-fold. Also, Ruzov et al [27] observes that expressing MLH1 causes apoptosis in HCT116 cells as well as during the onset of gastrulation in embryos.

The mechanism by which MLH1 induces apoptosis thereby protecting against tumor formation is not fully understood. There are several studies that establish the c-Abl protein as a mediator in the MLH1-dependent cellular response to damage [16, 28-30]. Therefore in this study, we investigated whether c-Abl plays a crucial role in the MLH1-induced apoptotic response in PCa cells. We demonstrate phosphorylation of c-Abl to be a key component in MLH1-triggered apoptosis in DU145 cells as knockdown of c-Abl with the inhibitor STI571 abrogated the apoptotic effect in these cells. Also, the phosphorylated form of c-Abl is necessary as no differences were detected with the non-phosphorylated form between treatment groups. It is noteworthy to point out however, that although siMLH1 efficiently reduced MLH1 expression to pCMV levels, phosphorylated $\mathrm{c}-\mathrm{Abl}$ was reduced but not to the same effect. This may be due to the fact that siMLH1 treatment started during the growth phase and cells had already accumulated phosphorylated c-Abl to an extent. This is further corroborated by the observation that apoptosis was also dramatically reduced but not to that of pCMV levels by all three siMLH1 treatments. The phosphorylation of c-Abl is thus critical for its activation and has been shown to occur at tyrosine sites $[31,32]$. Further, our antibody detects phosphorylation at tyrosine-204 of the SH2 domain and studies show phosphorylation of this core region of c-Abl to impact protein conformation and signaling [33]. The process by which c-Abl is phosphorylated is not known. Studies have shown that activation of $\mathrm{c}-\mathrm{Abl}$ is dependent on the ataxia telangiectasia mutated (ATM) gene $[34,35]$. In a study by Baskaran et al [35], ATM was required to activate $\mathrm{c}-\mathrm{Abl}$ and was due to phosphorylation at serine-465. Interestingly, a feedback for activation between these molecules was also observed [36].

The dependence of c-Abl in the MLH1 signaling pathway is in agreement with others as $\mathrm{Li}$ et al [16] observed MLH1-expressing cells to cause apoptosis in a p53-independent manner but when treating cells with STI571 or creating stable c-Abl knockdown transfectants, MLH1-dependent apoptosis was prevented. These authors further show the MLH1/c-Abl downstream effectors p $73 \alpha$ and GADD $45 \alpha$ to be of importance in the apoptotic pathway. In a report by Kim et al [29], MLH1 was also found to trigger the MEKK-1/MKK4/JNK/c-jun pathway in response to DNA damage. Interestingly, they find that $\mathrm{c}-\mathrm{Abl}$ is required to activate this signaling cascade by MLH1. In fact, they observe a functional interacting complex between MLH1 and c-Abl. Thus although it is apparent that MLH1 affects alternative signaling pathways, evidence strongly suggests that $\mathrm{c}-\mathrm{Abl}$ is a critical mediator for the MLH1-induced response.

In summary, this is the first report that shows MLH1 to display a protection against PCa by inhibiting cell proliferation, migration, and invasion in vitro as well as tumor growth in vivo. MLH1 induced apoptosis and was dependent on phosphorylation of c-Abl. Thus, we conclude that phosphorylated c-Abl plays a key role in the tumor suppressive effect of the MLH1 signaling pathway in prostate cells.

\section{MATERIALS AND METHODS}

\section{Cell lines and reagents}

Human PCa cell line DU145 and non-malignant epithelial prostate cell lines PWR-1E and RWPE-1 were purchased from American Type Culture Collection (Manassas, VA). Keratinocyte serum-free medium, bovine pituitary extract and human recombinant epidermal growth factor were purchased from Invitrogen (Carlsbad, CA). RPMI 1640, Opti-minimum essential medium and penicillin/streptomycin were obtained from the UCSF Cell Culture Facility (San Francisco, CA). Fetal bovine serum (FBS) was a product of Atlanta Biologicals (Lawrenceville, GA).

\section{Cell culture}

DU145 cells were cultured in RPMI 1640 medium supplemented with $10 \%$ FBS. PWR-1E and RWPE1 cells were cultured in keratinocyte growth medium supplemented with $5 \mathrm{ng} / \mathrm{mL}$ human recombinant epidermal growth factor and $0.05 \mathrm{mg} / \mathrm{mL}$ bovine pituitary extract. All cell lines were maintained at $37^{\circ} \mathrm{C}$ in a humidified 
atmosphere composed of $5 \% \mathrm{CO}_{2}$ and $95 \%$ air.

\section{Western blot analysis}

Whole cell extracts from cultured cells were prepared using radioimmunoprecipitation assay buffer (Thermo Scientific, Rockford, IL) containing protease inhibitor cocktail (Roche Diagnostics, Basel, Switzerland). Protein quantification was done using a BCA protein assay kit (Thermo Scientific) according to the manufacturer's instructions. Total protein $(20 \mu \mathrm{g})$ was loaded onto $4-12 \%$ bis-tris gels with 3 - $(N$-morpholino) propanesulfonic acid buffer and separated by a NuPAGE electrophoresis system (Invitrogen). Protein was transferred to Invitrogen ${ }^{\mathrm{TM}}$ polyvinylidene difluoride and immunoblotting was carried out according to standard protocols. Monoclonal antibodies against MLH1, cleaved PARP, c-Abl, and phosphorylated c-Abl were purchased from Cell Signaling (Danvers, MA) and monoclonal antibody against GAPDH (Santa Cruz Biotechnology, Santa Cruz, CA) was used to confirm equal loading. The membrane was washed and then incubated with secondary antibodies conjugated to horseradish peroxidase (Cell Signaling Technology). Protein complexes were visualized with the Echo-chemiluminescence (ECL) Detection System (GE Healthcare, Little Chalfont, UK) using the Chemidoc Imaging System (Bio-Rad Laboratories, Hercules, CA).

\section{RNA extraction}

Total RNA was extracted from DU145, PWR$1 \mathrm{E}$ and RWPE-1 cells using the RNeasy Mini kit (Qiagen, Valencia, CA) according to the manufacturer's instructions.

\section{Quantitative reverse transcription-polymerase chain reaction}

Extracted RNA was reverse-transcribed into complementary DNA (cDNA) using iScript cDNA Synthesis kit (Bio-Rad) and TaqMan MicroRNA Reverse Transcription kit (Applied Biosystems, Foster City, CA). Quantitative real-time PCR analysis was performed with an Applied Biosystems Prism 7500 Fast Sequence Detection System using TaqMan Universal PCR master mix according to the manufacturer's protocol (Applied Biosystems). Levels of RNA expression were determined using the 7500 Fast System SDS software version 1.3.1 (Applied Biosystems). PCR parameters for cycling were as follows: $95^{\circ} \mathrm{C}$ for 20 seconds, then 40 cycles of $95^{\circ} \mathrm{C}$ for 3 seconds and $60^{\circ} \mathrm{C}$ for 30 seconds. All reactions were done in a $10 \mu \mathrm{L}$ reaction volume in triplicate. The data were analyzed using the delta-delta $\mathrm{Ct}$ method to calculate the fold-change. TaqMan probes and primers for MLH1 (assay ID: Hs00179866_m1) and GAPDH (assay ID: Hs02758991_g1) were obtained from Applied Biosystems. GAPDH was used as internal control.

\section{Establishment of DU145 cells stably expressing MLH1}

DU145 cells were transfected with pCMV6-ENTRY vector expressing the C-terminally Myc and Flag-tagged human MLH1 cDNA as well as empty pCMV6-ENTRY vector as a control (OriGene Techologies, Rockville, MD) using X-treme Gene HD transfection reagent (Roche Diagnostics, Indianapolis, IN) according to the manufacturer's protocol. Clones were selected using 500 $\mu \mathrm{g} / \mathrm{ml}$ of G418 (Invitrogen). Colonies resistant to G418 appeared within 2 weeks and single colonies were picked and then expanded for another 3 weeks to make stable clone stock cells.

\section{Cell proliferation assay}

An MTS based assay was utilized to determine cell proliferation. Cells were plated in triplicate in 96well microplates at a density of $5 \times 10^{3}$ cells per well. At the desired time point, the number of viable cells was determined by adding CellTiter 96 AQueous One Solution reagent (Promega, Madison, WI) to each well and measuring the absorbance at $490 \mathrm{~nm}$ on a SpectraMax 190 plate reader (Molecular Devices, Sunnyvale, CA). Results were expressed as the percentage of optical density with absorbance of control cells being 100\%.

\section{Migration and Invasion assays}

Cell migration was evaluated by a wound-healing assay. Cells were plated in six-well dishes and monolayers were scraped using a P-20 micropipette tip. The width of the initial gap ( 0 hour) and the residual gap 24 hours after wounding were calculated from photomicrographs taken using a Nikon Eclipse TS100 microscope (Technical Instruments, Burlingame CA). Cell invasion properties were measured by a procedure using the CytoSelect Invasion assay (Cell Biolabs, San Diego CA) along with modified Boyden chambers consisting of transwellprecoated Matrigel membrane filter inserts with eight micron pores in 24-well tissue culture plates (BD Biosciences, Bedford, MA). Minimum essential medium containing $10 \% \mathrm{FBS}$ in the lower chamber served as the chemo-attractant. After 24 hours, invasive cells were stained and observed by photomicrographs taken with the Nikon microscope and quantified using the SpectraMax plate reader at absorbance of $560 \mathrm{~nm}$. 


\section{In vivo tumor growth}

All animal care was in accordance with the guidelines and this study was approved by the San Francisco Veterans Affairs IACUC (Institutional Animal Care and Use Committee). Animal users completed training programs to handle and work with mice through AALAS (American Association for Laboratory Animal Science) prior to animal experiments. For the subcutaneous xenograft mouse model, DU145 cells (5 x $10^{6}$ ) stably transfected with MLH1 or empty pCMV6ENTRY vector were suspended in $100 \mu \mathrm{L}$ RPMI 1640 medium and subcutaneously injected into the right backside flank of five week old female nude mice (strain BALB/c nu/nu; Charles River Laboratories, Wilmington, MA). Five nude mice were used per treatment group and tumor growth was examined over the course of 35 days. Tumor volume was calculated on the basis of width (x) and length (y) using the formula: $x^{2} y / 2$, where $x<y$.

\section{Apoptosis assay}

Apoptosis was analyzed with an annexin V-fluorescein isothiocyanate (FITC)/7-amino-actinomycin D staining system obtained from BD Biosciences (San Diego, CA). Briefly, prostate cells were harvested and resuspended in binding buffer at a concentration of $1 \times 10^{6}$ cells $/ \mathrm{ml}$. For each assay, $1 \times 10^{5}$ cells were incubated with $5 \mu \mathrm{l}$ of annexin V-FITC and $5 \mu \mathrm{l}$ of 7-amino-actinomycin $\mathrm{D}$ in the dark for $15 \mathrm{~min}$ at room temperature. After adding $400 \mu 1$ of binding buffer, samples were analyzed within an hour by a Cell Lab Quanta ${ }^{\mathrm{TM}}$ SC MPL flow cytometer (Beckman Coulter, Fullerton, CA).

\section{Small interfering RNA transfection}

Cells grown to $30-50 \%$ confluence were treated with three different sets of small interfering RNA (siRNA) duplexes specific for human MLH1 (siMLH1) or universal scrambled negative control (OriGene Techologies) by transfection using Lipofectamine 2000 transfection reagent (Invitrogen) as described by the manufacturer. Knockdown efficiency was evaluated by both realtime PCR and Western blotting for the three siMLH1s at different concentrations and the optimal condition selected was $30 \mathrm{nM}$. Vector control cells treated with Lipofectamine alone was also included.

\section{STI571 treatment}

pCMV and MLH1-transfected DU145 cells were grown in six-well plates. After two days, cells were treated with either $10 \mu \mathrm{M}$ STI571 (via Fisher Scientific, Pittsburg PA) or dimethylsulfoxide (DMSO) diluent (Sigma-
Aldrich, St Louis MO). Cells were then allowed to grow for another two days and harvested to determine apoptosis as described above.

\section{Statistical analysis}

Values in Figures are presented as the mean \pm standard error of mean (SEM) based on results obtained from at least three independent experiments. For in vivo studies, results are based on five animals per group. The relationship between variables was analyzed using the non-parametric Mann-Whitney U test or two-tailed Student's t-test. All analyses were performed using Expert StatView (version 4, SAS Institute, Cary, NC).

\section{ACKNOWLEDGEMENTS}

The authors declare no conflict of interests. This work was supported by the Veterans Affairs Merit Review and Program Project awards.

\section{REFERENCES}

1. Siegel R, Ma J, Zou Z, Jemal A. Cancer statistics, 2014. CA Cancer J Clin. 2014; 64:9-29.

2. Buermeyer AB, Deschenes SM, Baker SM, Liskay RM. Mammalian DNA mismatch repair. Annu Rev Genet. 1999; 33:533-564.

3. Kunkel TA, Erie DA. DNA mismatch repair. Annu Rev Biochem. 2005; 74:681-710.

4. Kinzler KW, Vogelstein B. Cancer-susceptibility genes. Gatekeepers and caretakers. Nature. 1997; 386:761, 763.

5. Li GM. The role of mismatch repair in DNA damageinduced apoptosis. Oncol Res. 1999; 11:393-400.

6. O'Brien V, Brown R. Signalling cell cycle arrest and cell death through the MMR System. Carcinogenesis. 2006; 27:682-692.

7. Soravia $\mathrm{C}$, van der Klift $\mathrm{H}$, Brundler MA, Blouin JL, Wijnen J, Hutter P, Fodde R, Delozier-Blanchet C. Prostate cancer is part of the hereditary non-polyposis colorectal cancer (HNPCC) tumor spectrum. Am J Med Genet A. 2003; 121A:159-162.

8. Raymond VM, Mukherjee B, Wang F, Huang SC, Stoffel EM, Kastrinos F, Syngal S, Cooney KA, Gruber SB. Elevated risk of prostate cancer among men with Lynch syndrome. J Clin Oncol. 2013; 31:1713-1718.

9. Grindedal EM, Moller P, Eeles R, Stormorken AT, Bowitz-Lothe IM, Landro SM, Clark N, Kvale R, Shanley $\mathrm{S}$, Maehle L. Germ-line mutations in mismatch repair genes associated with prostate cancer. Cancer Epidemiol Biomarkers Prev. 2009; 18:2460-2467.

10. Peltomaki P, Vasen H. Mutations associated with HNPCC predisposition -- Update of ICG-HNPCC/INSiGHT mutation database. Dis Markers. 2004; 20:269-276. 
11. Boyer JC, Umar A, Risinger JI, Lipford JR, Kane M, Yin S, Barrett JC, Kolodner RD, Kunkel TA. Microsatellite instability, mismatch repair deficiency, and genetic defects in human cancer cell lines. Cancer Res. 1995; 55:60636070 .

12. Chen Y, Wang J, Fraig MM, Metcalf J, Turner WR, Bissada NK, Watson DK, Schweinfest CW. Defects of DNA mismatch repair in human prostate cancer. Cancer Res. 2001; 61:4112-4121.

13. Yeh CC, Lee C, Dahiya R. DNA mismatch repair enzyme activity and gene expression in prostate cancer. Biochem Biophys Res Commun. 2001; 285:409-413.

14. Chen Y, Wang J, Fraig MM, Henderson K, Bissada NK, Watson DK, Schweinfest CW. Alterations in PMS2, MSH2 and MLH1 expression in human prostate cancer. Int J Oncol. 2003; 22:1033-1043.

15. Soni A, Bansal A, Singh LC, Mishra AK, Majumdar M, Regina T, Mohanty NK, Saxena S. Gene expression profile and mutational analysis of DNA mismatch repair genes in carcinoma prostate in Indian population. OMICS. 2011; $15: 319-324$

16. Li LS, Morales JC, Hwang A, Wagner MW, Boothman DA. DNA mismatch repair-dependent activation of c-Abl/ p73alpha/GADD45alpha-mediated apoptosis. J Biol Chem. 2008; 283:21394-21403.

17. Fink D, Nebel S, Aebi S, Zheng H, Cenni B, Nehme A, Christen RD, Howell SB. The role of DNA mismatch repair in platinum drug resistance. Cancer Res. 1996; 56:48814886.

18. Papouli E, Cejka P, Jiricny J. Dependence of the cytotoxicity of DNA-damaging agents on the mismatch repair status of human cells. Cancer Res. 2004; 64:33913394.

19. Zhang H, Richards B, Wilson T, Lloyd M, Cranston A, Thorburn A, Fishel R, Meuth M. Apoptosis induced by overexpression of hMSH2 or hMLH1. Cancer Res. 1999; 59:3021-3027.

20. Kolodner RD, Hall NR, Lipford J, Kane MF, Morrison PT, Finan PJ, Burn J, Chapman P, Earabino C, Merchant E, Bishop DT. Structure of the human MLH1 locus and analysis of a large hereditary nonpolyposis colorectal carcinoma kindred for mlh1 mutations. Cancer Res. 1995; 55:242-248.

21. Tewari M, Quan LT, O'Rourke K, Desnoyers S, Zeng Z, Beidler DR, Poirier GG, Salvesen GS, Dixit VM. Yama/ CPP32 beta, a mammalian homolog of CED-3, is a CrmA-inhibitable protease that cleaves the death substrate poly(ADP-ribose) polymerase. Cell. 1995; 81:801-809.

22. Margolin N, Raybuck SA, Wilson KP, Chen W, Fox T, $\mathrm{Gu}$ Y, Livingston DJ. Substrate and inhibitor specificity of interleukin-1 beta-converting enzyme and related caspases. J Biol Chem. 1997; 272:7223-7228.

23. Hassen S, Ali N, Chowdhury P. Molecular signaling mechanisms of apoptosis in hereditary non-polyposis colorectal cancer. World J Gastrointest Pathophysiol. 2012; 3:71-79.

24. Koi M, Umar A, Chauhan DP, Cherian SP, Carethers JM, Kunkel TA, Boland CR. Human chromosome 3 corrects mismatch repair deficiency and microsatellite instability and reduces $\mathrm{N}$-methyl-N'-nitro-N-nitrosoguanidine tolerance in colon tumor cells with homozygous hMLH1 mutation. Cancer Res. 1994; 54:4308-4312.

25. Hawn MT, Umar A, Carethers JM, Marra G, Kunkel TA, Boland CR, Koi M. Evidence for a connection between the mismatch repair system and the G2 cell cycle checkpoint. Cancer Res. 1995; 55:3721-3725.

26. Ding $X$, Mohd AB, Huang $Z$, Baba T, Bernardini MQ, Lyerly HK, Berchuck A, Murphy SK, Buermeyer AB, Devi GR. MLH1 expression sensitises ovarian cancer cells to cell death mediated by XIAP inhibition. Br J Cancer. 2009; 101:269-277.

27. Ruzov A, Shorning B, Mortusewicz O, Dunican DS, Leonhardt H, Meehan RR. MBD4 and MLH1 are required for apoptotic induction in xDNMT1-depleted embryos. Development. 2009; 136:2277-2286.

28. Nehme A, Baskaran R, Nebel S, Fink D, Howell SB, Wang $\mathrm{JY}$, Christen RD. Induction of JNK and c-Abl signalling by cisplatin and oxaliplatin in mismatch repair-proficient and -deficient cells. Br J Cancer. 1999; 79:1104-1110.

29. Kim WJ, Rajasekaran B, Brown KD. MLH1- and ATMdependent MAPK signaling is activated through c-Abl in response to the alkylator N-methyl-N'-nitro-N'nitrosoguanidine. J Biol Chem. 2007; 282:32021-32031.

30. Wagner MW, Li LS, Morales JC, Galindo CL, Garner HR, Bornmann WG, Boothman DA. Role of c-Abl kinase in DNA mismatch repair-dependent G2 cell cycle checkpoint arrest responses. J Biol Chem. 2008; 283:21382-21393.

31. Hantschel O, Superti-Furga G. Regulation of the c-Abl and Bcr-Abl tyrosine kinases. Nat Rev Mol Cell Biol. 2004; 5:33-44.

32. Chen S, O'Reilly LP, Smithgall TE, Engen JR. Tyrosine phosphorylation in the $\mathrm{SH} 3$ domain disrupts negative regulatory interactions within the c-Abl kinase core. J Mol Biol. 2008; 383:414-423.

33. Meyn MA, 3rd, Wilson MB, Abdi FA, Fahey N, Schiavone AP, Wu J, Hochrein JM, Engen JR, Smithgall TE. Src family kinases phosphorylate the Bcr-Abl SH3-SH2 region and modulate Bcr-Abl transforming activity. J Biol Chem. 2006; 281:30907-30916.

34. Shafman T, Khanna KK, Kedar P, Spring K, Kozlov S, Yen T, Hobson K, Gatei M, Zhang N, Watters D, Egerton M, Shiloh Y, Kharbanda S, Kufe D, Lavin MF. Interaction between ATM protein and c-Abl in response to DNA damage. Nature. 1997; 387:520-523.

35. Baskaran R, Wood LD, Whitaker LL, Canman CE, Morgan $\mathrm{SE}, \mathrm{Xu} \mathrm{Y}$, Barlow C, Baltimore D, Wynshaw-Boris A, Kastan MB, Wang JY. Ataxia telangiectasia mutant protein activates c-Abl tyrosine kinase in response to ionizing 
radiation. Nature. 1997; 387:516-519.

36. Wang X, Zeng L, Wang J, Chau JF, Lai KP, Jia D, Poonepalli A, Hande MP, Liu H, He G, He L, Li B. A positive role for $\mathrm{c}-\mathrm{Abl}$ in Atm and Atr activation in DNA damage response. Cell Death Differ. 2011; 18:5-15. 